\title{
Questões acerca da percepção sonora de harmônicos: a função das variáveis sexo e aprendizagem
}

\author{
Matters concerning sound perception of harmonics: \\ the function of the sex and apprenticeship variables
}

\author{
Deborah Christina ANTUNES \\ Amauri GOUVEIA Jr2
}

\begin{abstract}
Resumo
Este trabalho objetivou verificar o desempenho de homens e mulheres em uma tarefa de discriminação perceptiva de sons e o efeito de aprendizagem anterior sobre esta discriminação. O experimento foi realizado com 17 homens e 17 mulheres, submetidos individualmente a uma sessão de coleta de dados composta de questionário padronizado e de teste de discriminação com vinte tentativas gravadas em um CD-ROM. A análise estatística dos dados indicou diferenças de desempenho entre pessoas com e sem experiência musical prévia $[F(1,33)=14,69, p<0,001]$. Embora não significativo, as mulheres apresentaram melhor desempenho no grupo sem experiência prévia, e no grupo com experiência, os homens apresentaram melhor desempenho. Os dados indicam que, embora haja diferenças sexuais na percepção sonora, a experiência parece ser o fator mais importante neste tipo de discriminação.
\end{abstract}

Unitermos: Aprendizagem. Diferenças sexuais humanas. Discriminação perceptiva. Percepção musical.

\begin{abstract}
The aim of this study was to verify the performances of men and women in a discriminative test of sound harmonics perception, and the effect of prior learning on this discrimination. The experiment was conducted using 17 men and 17 women, who were individually submitted to an experimental session with a standard questionnaire and a sound perception test comprising 20 attempts recorded on a CD-ROM. The statistical analysis of the data shows that subjects, both with and without experience of learning a musical instrument, have a difference in performance in the test $[F(1.33)=14.69, p<0.001]$. Though not significant, women displayed a better performance in the group without learning, and in the group with experience, men showed better performance. The data show that, though there may be differences between the genders in terms of sound perception, previous experience is ostensibly the most important factor in this type of discrimination.
\end{abstract}

Uniterms: Learning. Gender differences. Perceptual discrimination. Musical perception.

Questões acerca das habilidades humanas, tais como se seriam inatas ou adquiridas ou se haveriam diferenças entre os sexos, são usualmente correntes, não apenas em ambientes cotidianos com opiniões advindas do senso comum, mas também no ambiente acadêmico, gerando discussões e controvérsias. Rohden

$\nabla \nabla \nabla \nabla \nabla$

1 Universidade Federal de São Carlos, Programa de Pós-Graduação em Filosofia. Rod. Washington Luís, Km235, SP 310, São Carlos, SP, Brasil. Correspondência para/Correspondence to: D. C. ANTUNES. E-mail: <deborahantunes@yahoo.com.br>.

2 Universidade Federal do Pará, Centro de Ciências Biológicas, Departamento de Fisiologia. Belém, PA, Brasil. 
(2003) realizou um estudo de revisão e análise histórico-filosófica a respeito da construção da diferença sexual na medicina do século XIX. A autora baseou-se nas produções acadêmicas da área no período e no estudo das teorias da ciência do século XVIII que, com a influência newtoniana e as transformações econômicas e políticas, passou a trabalhar com um modelo no qual existiam dois sexos radicalmente distintos, diferentemente da herança grega que a influenciava até então. Segundo Rohden (2003), tais produções estão repletas do que chamou de naturalização de diferenças, tais como vulnerabilidade física, moral e intelectual, e argumentações sobre sua imutabilidade independentemente das influências externas, no sentido de defender certa fragilidade permanente nos sujeitos do sexo feminino.

Na área de psicologia, diversos trabalhos tratam das diferenças perceptuais entre homens e mulheres (Becker, 1993; Beech \& Beauvois, 2005; Breedlove, 1993; Hampson \& Kimura, 1993; Jongman, Sereno \& Wang, 2001; Leontiev, 1960; Nater, Abbruzzese, Krebs \& Ehlert, 2006; Sanders \& Wenmoth, 1998; Sluming \& Manning, 2000). Alguns destes atribuem mais valor às variáveis biológicas, como os efeitos hormonais (Becker, 1993; Beech \& Beauvois, 2005; Breedlove, 1993; Sanders \& Wenmoth, 1998; Sluming \& Manning, 2000); outros, às variáveis ambientais, como a socialização e a aprendizagem (Jogman et al., 2001; Leontiev, 1960). Eagly e Wood (1999) apresentam duas teorias divergentes sobre a origem das diferenças sexuais no comportamento humano, dentro das quais parecem estar presentes os trabalhos citados. A primeira envolve disposições biológicas e baseia-se em uma psicologia evolucionária segundo a qual adaptações evolutivas, por meio de processos que envolvem mecanismos específicos, causaram diferenças psicológicas e sexuais. Assim, seria devido a tal distinção que homens e mulheres diferem psicologicamente e ocupam diferentes posições sociais. A segunda busca respostas nas estruturas sociais, e é representada pela psicologia social. De acordo com os autores citados, essa teoria defende que o fato de homens e mulheres ocuparem diferentes posições sociais torna-os psicologicamente diferentes, de modo a se ajustarem a essas posições.

Pesquisas mostram que, de fato, homens e muIheres apresentam diferentes desempenhos em tarefas que requerem habilidades distintas (Flores-Mendoza,
2000; Gil-Verora et al., 2003; Rosa e Silva \& Sá, 2006). Assim, além das diferenças anatômicas externas e dos caracteres sexuais primários e secundários, sabe-se que existem várias outras diferenças de desempenho em funções como a linguagem (Flores-Mendoza, 2000; Hampson \& Kimura, 1993) o processamento das informações, as emoções, as sensações (Gil-Verora et al., 2003; Hampson \& Kimura, 1993), a cognição em geral e o humor (Rosa e Silva \& Sá, 2006). Ou seja, de acordo com tais autores, as diferenças estão nas maneiras pelas quais os cérebros dos homens e das mulheres funcionam, e como isto se reflete no comportamento e na cognição, embora muitas destas diferenças sejam sutis.

Segundo Hampson e Kimura (1993), em resultados de testes padronizados de QI não há diferença no desempenho geral entre os sexos, mas sim, no desempenho em habilidades específicas. No caso, os homens teriam desempenho melhor em atividades que requerem habilidades espaciais, quantitativas e de força, e as mulheres em atividades que requisitam habilidades verbais, de velocidade perceptual e acurácia, bem como coordenação motora fina. Segundo os autores, tais diferenças manifestam-se desde alguns meses após o nascimento, o que sugere um determinante biológico, uma vez que o contato com a cultura e a possibilidade de aprendizagem ainda não foram muito extensos nesta fase.

Becker (1993) afirma que é possível que o efeito de hormônios esteroides no cérebro seja um fator importante nos comportamentos motores e sensório-perceptuais. Sabe-se que a exposição pré-natal aos hormônios desencadeia efeitos organizacionais permanentes, enquanto flutuações hormonais periódicas no adulto produzem efeitos de ativação no cérebro e no comportamento (Sanders \& Wenmoth, 1998). A primeira é a chamada ação organizadora e ocorre na vida intrauterina; a segunda é a ação ativacional e ocorre na vida adulta (Breedlove, 1993). Nesse mesmo sentido, Sluming e Manning (2000) afirmam que o nível de testosterona ao qual o feto é exposto no período pré-natal leva a uma lateralização do sistema dopaminérgico, o que facilita o desenvolvimento do hemisfério direito, realçando habilidades como matemática, espacial e musical. No caso, a exposição do feto à testosterona teria uma ação organizadora para a aptidão a tais habilidades. 
Atendo-se a esta questão, Sluming e Manning (2000) afirmam que a música humana seria explicável em termos de seleção sexual. Desta forma, homens e mulheres que tenham sido expostos a um maior nível de testosterona durante a gestação tenderiam a ter realçadas habilidades musicais. No entanto, isto não significa que necessariamente tais habilidades sejam mais acuradas em homens, já que o julgamento da boa música, em particular, seria crucial em qualquer situação de corte; assim, seria mais apurado quando a mulher está em seu período fértil e, neste caso, a ação hormonal teria um efeito ativacional. O desempenho de escuta das mulheres parece depender da fase do ciclo menstrual, isto porque uma mudança na interpretação musical do hemisfério esquerdo para o hemisfério direito parece ser favorável quando o nível de estrogênio está baixo, o que é possível na ovulação, que é associada à baixa concentração de estrogênio - e esta se correlaciona com o realce da apreciação musical.

Porém, para Sanders e Wenmoth (1998) o que ocorre é justamente o contrário. Embora também aqui os efeitos ativacionais dos hormônios na assimetria cerebral e na cognição sejam discutidos em relação às diferenças sexuais, os autores relatam que o desempenho auditivo das mulheres, em um teste de identificação de vozes consonantes e em outro de reconhecimento de coro musical, modifica-se em diferentes fases do ciclo menstrual. O que ocorre é uma diminuição no desempenho do ouvido esquerdo (hemisfério direito) e aumento do desempenho do ouvido direito (hemisfério esquerdo) durante o período fértil. Deste modo, pode-se notar que há, na literatura especializada, discordâncias em relação às habilidades perceptuais no que tange aos desempenhos de homens e de mulheres, e na ação hormonal correlata.

Por outro lado, representantes do que Eagly e Wood (1999) chamaram de teoria de origem baseada na psicologia social também realizaram estudos a respeito da percepção sonora, embora poucos tenham sido encontrados. Um deles é um estudo clássico de Leontiev (1960), no qual foram realizados experimentos visando demonstrar que diferenças existentes na percepção de sons (tons) ocorrem de acordo com a experiência vivida por cada indivíduo em relação a eles, seja por diferenças culturais (como em idiomas tonais), ou de atividade ocupacional em relação ao som. Outro exemplo, mais atual, é a pesquisa de Jongman et al. (2001), na qual os autores compararam a escuta de chineses e americanos de tons mandarins, verificando que nos chineses havia uma vantagem do ouvido direito (hemisfério esquerdo) sobre o esquerdo (hemisfério direito), enquanto nos americanos (sem experiência com linguagem tonal) tal vantagem não existiu. Segundo tais estudos, a aprendizagem não apenas interfere na percepção sonora, como tem um papel fundamental nela.

Enquanto alguns pesquisadores focam suas pesquisas e análises na questão biológica, procurando verificar a influência hormonal no desempenho de atividades realizadas por homens e mulheres, outros buscam explicações no ambiente e no papel da aprendizagem, embora não tenham realizado pesquisas em relação às diferenças entre homens e mulheres, mas entre pessoas de países e etnias diferentes. Dentre os primeiros, no que tange à habilidade de percepção musical, há ainda controvérsias; por exemplo, sobre o efeito ativacional dos hormônios em mulheres durante a ovulação para uma melhora em tal percepção e sobre o efeito organizacional na vida intrauterina que definiria uma habilidade perceptual mais acurada. Já os segundos estão em concordância sobre a função essencial exercida pela aprendizagem no desempenho em atividades que requerem tais habilidades. Tendo em vista a divergência de dados na literatura a respeito da discriminação sonora, o objetivo desta pesquisa foi estudar se existem diferenças no desempenho de uma tarefa de discriminação de sons harmônicos, entre homens e mulheres, com e sem história prévia de aprendizagem no contato com instrumentos musicais.

\section{Método}

\section{Participantes}

Participaram do estudo 34 pessoas; 17 eram homens (média de idade (M) 20,53, desvio-padrão - $d p=3,37$ ) e 17 eram mulheres $(M=20,00, d p=2,40)$.

\section{Instrumentos}

Para a realização da pesquisa foram utilizados: um questionário, um CD-ROM contendo os estímulos 
do teste, um discman (Kenwood portable compact disc player, dpc-171), um fone de ouvido (Sontec CD-128 - frequência: 20-18,000HZ, impedância: 32ohm, potência máxima de entrada: $100 \mathrm{mw}$ ), folha de respostas desenvolvida para o teste, régua e caneta.

O questionário continha as seguintes questões: nome, sexo, idade, data de nascimento, escolaridade, profissão, com que frequência ouve música, qual o tipo de música preferido, se toca algum instrumento (com que frequência e se é profissionalmente ou não), se realizou algum curso de música, se faz uso de algum medicamento ou droga ou o fez nos últimos três meses, se é destro ou canhoto, se possui ciclo menstrual regular (no caso das mulheres), qual a data da última menstruação (no caso das mulheres).

O CD-ROM tinha 23 faixas. A primeira continha as instruções a respeito do teste propriamente dito, exatamente como segue:"Cada faixa deste é composta por cinco sons diferentes e cinco sons iguais que estão entre um som diferente e outro. O primeiro som é o modelo; você deve optar por um dos quatro sons diferentes subsequentes, podendo escolher até dois sons em caso de dúvida. Não há resposta errada. Você deve marcar sua opção na folha de respostas assinalando qual o som escolhido e depois entregá-la ao experimentador".

As duas faixas seguintes consistiram em tentativas para o participante verificar se havia entendido as instruções, e as vinte seguintes consistiam no teste propriamente dito. Cada faixa do CD-ROM, a partir da segunda, continha um estímulo modelo, em seguida um estímulo que foi chamado de "som branco" (explicado a seguir); após o som branco, o primeiro estímulo-resposta e, em seguida, outro som branco similar ao primeiro; foram quatro estímulos de comparação (estímulos-respostas) em cada faixa intercalados com o som branco, conforme exemplo na Figura 1.

Os estímulos-modelo foram terças e quintas harmônicas de notas musicais retiradas das duas oitavas centrais do piano: do-mi, do-sol, re-fa\#, re-la, mi-sol\#, misi, fa-la, fa-do, sol-si, sol-re, la-do, la-mi, si-ré\#, si-fá\#, do-mi, do-sol, re-fa\#, re-la, mi-sol\#, mi-si, fa-la, sol-si.

Os estímulos de comparação foram: um estímulo igual ao modelo, a terça (ou quinta) do modelo apresentado mais sua oitava, a primeira nota do estímulo apresentado mais sua oitava e as notas do estímulomodelo invertidas (na sua ordem). Cada estímulo foi intercalado com o "som branco", que equivale às notas das duas oitavas centrais do piano (de dó a dó) tocadas temporalmente juntas, a fim de evitar que o estímulo anterior interferisse na percepção do posterior. A ordem das faixas foi aleatorizada, bem como a ordem de apresentação dos estímulos de comparação.

As folhas de respostas continham as tentativas (faixas), e em cada tentativa quatro alternativas ( $A, B, C$ e D). Um exemplo da folha de respostas pode ser observado na Figura 2.

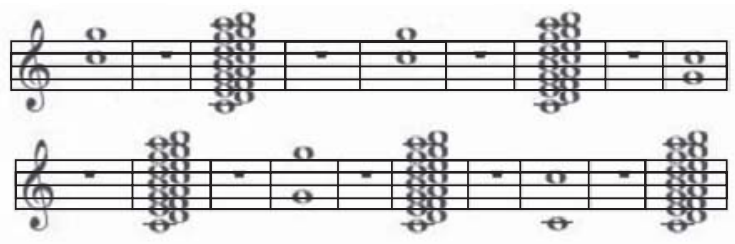

Figura 1. Exemplo de uma faixa do CD-ROM contendo: um estímulo modelo seguido de um som branco, do primeiro estímulo de comparação, outro som branco, do segundo estímulo de comparação, outro som branco, do terceiro estímulo de comparação, outro som branco, do quarto estímulo de comparação, outro som branco e fim da faixa.

\begin{tabular}{|c|c|c|c|c|}
\hline \multicolumn{5}{|c|}{ Folha de respostas } \\
\hline \multirow[t]{4}{*}{ Nome: } & & & & Data: \\
\hline & Exemplos: & & & \\
\hline & $1-A()$ & $B()$ & $C()$ & $D()$ \\
\hline & $2-A()$ & $B()$ & $C()$ & $D()$ \\
\hline $3-A()$ & $B()$ & $C()$ & $D()$ & \\
\hline $4-A()$ & $B()$ & $C()$ & $D()$ & \\
\hline $5-A()$ & $B()$ & $C()$ & $D()$ & \\
\hline $6-A()$ & $B()$ & $C()$ & $D()$ & \\
\hline $7-A()$ & $B()$ & $C()$ & $D()$ & \\
\hline $8-A()$ & $B()$ & $C()$ & $D()$ & \\
\hline $9-A()$ & $B()$ & $C()$ & $D()$ & \\
\hline $10-A()$ & $B()$ & $C()$ & $D()$ & \\
\hline $11-A()$ & $B()$ & $C()$ & $D()$ & \\
\hline $12-A()$ & $B()$ & $C()$ & $D()$ & \\
\hline $13-A()$ & $B()$ & $C()$ & $D()$ & \\
\hline $14-A()$ & $B()$ & $C()$ & $D()$ & \\
\hline $15-A()$ & $B()$ & $C()$ & $D()$ & \\
\hline $16-A()$ & $B()$ & $C()$ & $D()$ & \\
\hline $17-A()$ & $B()$ & $C()$ & $D()$ & \\
\hline $18-A()$ & $B()$ & $C()$ & $D()$ & \\
\hline $19-A()$ & $B()$ & $C()$ & $D()$ & \\
\hline $20-A()$ & $B()$ & $C()$ & $D()$ & \\
\hline $21-A()$ & $B()$ & $C()$ & $D()$ & \\
\hline $22-A()$ & $B()$ & $C()$ & $D()$ & \\
\hline
\end{tabular}

Figura 2. Folha de respostas com as tentativas e, em cada tentativa, quatro alternativas. Universidade Estadual Paulista Júlio de Mesquita Filho, campus Bauru (SP), 2003. 


\section{Procedimentos}

Todos os participantes responderam individualmente ao questionário inicial. Após esta fase cada um, em separado, ouviu as instruções contidas na primeira faixa do CD e os estímulos em uma única sessão e teste, marcando na folha de respostas qual alternativa identificou como correta. A régua foi utilizada para ajudar o participante a seguir as alternativas na folha de respostas.

A tarefa de cada participante foi discriminar/ identificar qual dos estímulos de comparação equivalia ao estímulo-modelo. Ao participante era permitido modificar o volume do aparelho quando achasse necessário, porém não era permitido que voltasse qualquer uma das faixas. Foram considerados acertos as escolhas iguais ao modelo. Havendo duas escolhas feitas, se uma das escolhas fosse igual ao modelo, a resposta era considerada correta.

Os dados foram tabulados em planilhas de dados conforme as informações demográficas coletadas nos questionários, sexo e experiência com instrumentos musicais. Para o primeiro grupo de dados (demográficos), foram feitas correlações (Coeficiente de Correlação de Spearman) de forma a obter possíveis indícios de variação dos dados de forma harmônica entre si. Para as variáveis "sexo" e "experiência com instrumentos musicais", a análise foi feita estatisticamente por meio de uma análise de variância para ambas as variáveis simultaneamente (ANOVA de duas vias), de forma a tentar identificar se os grupos diferiam entre si em relação a cada uma destas variáveis (homens versus mulheres; pessoas com experiência versus pessoas sem experiência) e em sua interação (mulheres com experiência versus mulheres sem experiência; homens com experiência versus homens sem experiência). Os resultados foram considerados significativos quando apresentaram uma probabilidade menor que 0,05 $(p<0,05)$

\section{Resultados}

A partir do teste, pôde-se observar que a média de acertos dos homens foi de 12,47, com um erro-padrão de 4,88 e um desvio-padrão de 0,15, sendo que a média de acertos das mulheres foi de 14,58, com um erro-padrão de 3,92 e um desvio-padrão de 0,13. Os resultados individuais podem ser encontrados na Tabela 1.

Na Figura 3 observa-se que as mulheres que não apresentaram história prévia de aprendizagem obtiveram uma média de acertos de 12,50, e os homens, de

Tabela 1. Idade, pontuação e experiência musical de cada participante. Média, desvio-padrão (dp) e erro-padrão (ep) da idade, pontuação e experiência musical dos participantes. Universidade Estadual Paulista Júlio de Mesquita Filho, campus Bauru (SP), 2003.

\begin{tabular}{|c|c|c|c|c|c|c|c|}
\hline Mulher & Idade & Score & Experiência & Homem & Idade & Score & Experiência \\
\hline 1 & 21 & 10 & não & 1 & 23 & 18 & $\operatorname{sim}$ \\
\hline 2 & 19 & 18 & sim & 2 & 25 & 5 & não \\
\hline 3 & 23 & 13 & não & 3 & 20 & 5 & não \\
\hline 4 & 20 & 18 & $\operatorname{sim}$ & 4 & 20 & 14 & $\operatorname{sim}$ \\
\hline 5 & 20 & 14 & não & 5 & 22 & 18 & $\operatorname{sim}$ \\
\hline 6 & 19 & 2 & $\operatorname{sim}$ & 6 & 30 & 13 & não \\
\hline 7 & 26 & 18 & $\operatorname{sim}$ & 7 & 21 & 10 & $\operatorname{sim}$ \\
\hline 8 & 18 & 15 & $\operatorname{sim}$ & 8 & 20 & 15 & não \\
\hline 9 & 20 & 15 & $\operatorname{sim}$ & 9 & 20 & 7 & não \\
\hline 10 & 19 & 15 & $\operatorname{sim}$ & 10 & 16 & 10 & não \\
\hline 11 & 18 & 16 & $\operatorname{sim}$ & 11 & 18 & 6 & não \\
\hline 12 & 19 & 15 & $\operatorname{sim}$ & 12 & 19 & 11 & não \\
\hline 14 & 24 & 14 & $\operatorname{sim}$ & 14 & 17 & 20 & $\operatorname{sim}$ \\
\hline 15 & 20 & 18 & $\operatorname{sim}$ & 15 & 21 & 19 & $\operatorname{sim}$ \\
\hline 16 & 17 & 17 & $\operatorname{sim}$ & 16 & 22 & 11 & $\operatorname{sim}$ \\
\hline 17 & 17 & 13 & não & 17 & 18 & 15 & $\operatorname{sim}$ \\
\hline média & 20 & 14,58 & & média & 20,53 & 12,47 & \\
\hline$d p \sigma$ & 2,4 & 3,92 & & $d p \sigma$ & 3,37 & 4,88 & \\
\hline ep & 0,1 & 0,13 & & ep & 0,12 & 1,15 & \\
\hline
\end{tabular}




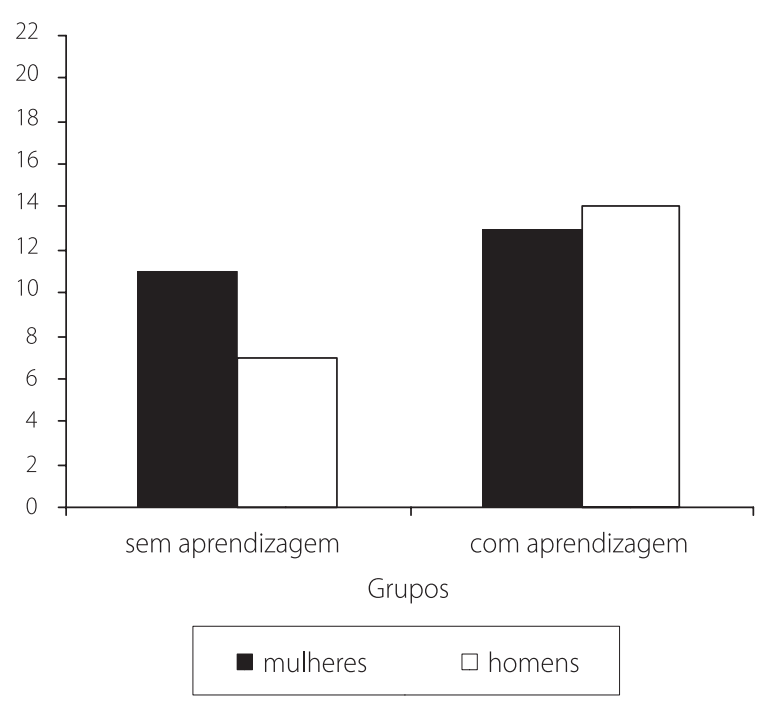

Figura 3. Pontuação dos participantes, divididos nos grupos mulheres e homens, sem e com aprendizagem. Universidade Estadual Paulista Júlio de Mesquita Filho, campus Bauru (SP), 2003

9,00 . As mulheres que relataram ter uma história prévia de aprendizagem musical obtiveram uma média de 15,23 , e os homens, de 15,56, de uma total de vinte pontos.

Nesta mesma figura pode-se perceber a diferença da média de acertos entre as mulheres com e sem aprendizagem e entre os homens, também com e sem aprendizagem. As outras variáveis coletadas por meio do questionário, tais como idade, escolaridade, profissão, frequência com que ouvia música, tipo de música preferido, uso de medicamento ou droga, se destro ou canhoto e, no caso das mulheres, período menstrual, quando comparadas estatisticamente com o resultado do teste, não apresentaram significância.

\section{Discussão}

Na introdução deste artigo foram apresentados trabalhos que divergem em relação à origem das diferenças perceptuais, se estritamente de fundo biológico ou social. Os resultados apresentados neste trabalho revelam que, no grupo que foi estudado, as mulheres apresentaram, de modo geral, um desempenho melhor em relação ao desempenho dos homens na tarefa de discriminação sonora de harmônicos proposta neste experimento, embora tal diferença não seja considerada estatisticamente significativa. A hipótese de que a percepção sonora dos homens é mais apurada do que a das mulheres devido à ação hormonal organizacional, e que a percepção das mulheres sofre flutuações de acordo com o período do ciclo menstrual, como afirmado por Sluming e Manning (2000), ou mesmo por Sanders e Wenmoth (1998), embora em direções opostas, parece não ter encontrado corres-pondência nos dados apresentados.

Talvez tal flutuação seja muito sutil para influenciar os resultados do teste aplicado, ou o tipo de tarefa proposto no experimento em questão dilua tal diferença. Isso não necessariamente significa que não haja influências de âmbito biológico na percepção de sons consonantes; a percepção é um processo complexo que inclui tanto a base do aparato biológico quanto a aprendizagem. De acordo com Forgus (1971), por percepção entendem-se os processos realizados pelo organismo, integrando em um único processo as informações do ambiente obtidas por meio dos órgãos sensoriais; deste modo, as percepções são a integração das sensações. Segundo Kandel, Schwartz e Jessell (1995), estas últimas consistem na recepção e transdução de eventos físicos do ambiente por meio de sistemas especializados para as diferentes modalidades sensoriais, capazes de filtrar propriedades relevantes dos estímulos, gerando dados para o sistema nervoso central. Na percepção, essas informações são transformadas em representações experienciadas pelo indivíduo como qualitativamente diversas (objetos, gostos, eventos, sons, figuras) e isso pode ocorrer de maneira simples ou exigir aprendizagem. Ou seja, o aparato perceptivo encontra-se pré-formado ao nascimento, mas é, em grande parte, influenciado posteriormente pela aprendizagem ao longo da história de vida (Forgus, 1971), que modela fatores como sensibilidade, preferência e discriminação de estímulos. Esta última, por sua vez, encontra-se entre os tipos de processos perceptuais mais complexos que envolvem aprendizagem (Forgus 1971; Roth \& Frisby, 1986). Segundo Matos (1981), ela ocorre quando um sujeito age de forma diferente diante de informações distintas do ambiente (estímulos).

No caso desse experimento, são diversos os estímulos que caracterizam um som complexo, como a fala (Fitch, Miller \& Tallal, 1997) e a música (Shamma, 1999), e influenciam diretamente sua percepção. São eles o tom, o timbre, a modulação, os intervalos e o 
volume (Shamma, 1999). Considera-se que os seres humanos são capazes de perceber coerentemente os atributos de cada fonte sonora que chega aos seus ouvidos, bem como julgá-los. Esta capacidade de analisar o som está baseada em um processo complexo descrito por Shamma (1999), no qual o som é primeiramente analisado em termos de algumas variáveis perceptualmente significantes e, em um nível mais avançado, tais variáveis são organizadas e agrupadas de acordo com regras específicas.

É interessante verificar, porém, como a variável "experiência prévia com instrumentos musicais" ou simplesmente "aprendizagem" sobressaiu em relação às variáveis "diferença sexual" e "período do ciclo menstrual", no caso das mulheres. Deste modo, a questão sobre a qual a análise deve pairar refere-se aos dados revelados pelo grupo dos sujeitos que relataram ter uma experiência prévia em relação aos instrumentos musicais, experiência esta que exige diretamente o desenvolvimento da percepção de sons harmônicos. Tal percepção, embora tenha um componente de base biológica, parece ter sido desenvolvida no decorrer da história de vida dos participantes. Esses sujeitos apresentaram um desempenho efetivamente superior àqueles que não tinham experiência em relação aos instrumentos musicais e à manipulação dos sons, independentemente se do sexo feminino ou masculino.

Assim como revelado por Leontiev (1960) e por Jongman et al. (2001), o fator cultural e a aprendizagem são os aspectos que mais influenciaram as habilidades que envolvem percepção sonora nesta tarefa. Ou seja, parece que, independentemente dos fatores referentes à lateralização e às flutuações hormonais, a aprendizagem foi o fator determinante para um desempenho superior. Assim, mesmo que existam diferenças garantidas pelo aparato biológico, favorecendo de imediato o desempenho de um sexo ou de outro, como algumas das pesquisas citadas na introdução afirmam, os dados apresentados permitem inferir que a aprendizagem é o fator determinante para o desempenho das habilidades de discriminação sonora de harmônicos, tal como foi realizada neste trabalho.

Resta, no entanto, questionar a dicotomia estabelecida entre as teorias explicitadas por Eagly e Wood (1999). Haveria, de fato, necessidade de opor variáveis biológicas e sociais? Tal oposição, tomada de um modo sectário, confere legitimidade à crítica de que cada uma contém em si um conteúdo ideológico influenciado pela própria história da ciência, tal como indicam as análises de Rohden (2003) no âmbito da construção das diferenças sexuais na medicina. Se o gênero humano participa, em seu desenvolvimento, de três níveis de seleção - filogenético, ontogenético e cultural -, reconhecer isso nos dados encontrados nos diversos experimentos científicos, de modo a tornar explícitas inclusive as contradições porventura encontradas, talvez traga mais legitimidade à própria ciência. Não obstante, mais trabalhos nesta direção parecem ser ainda necessários.

\section{Referências}

Becker, J. B. (1993). Hormonal influences on extrapyramidal sensoriomotor function and hippocampal plasticity. In J. B. Becker, S. M. Breedlove, D. Crews \& M. M. McCarthy (Eds.), Behavioral endocrinology (pp.325-356). Cambridge, Mass: MIT Press.

Beech, J. R., \& Beauvois, M. W. (2005). Early experience of sex hormones as a predictor of reading, phonology, and auditory perception. Brain and Language, 96, 49-58.

Breedlove, S. M. (1993). Sexual differentiation of the brain and behavior. In J. B. Becker, S. M. Breedlove, D. Crews \& M. M. McCarthy (Eds.), Behavioral endocrinology (pp.39-70). Cambridge, Mass: MIT Press.

Eagly, A. H., \&Wood, W. (1999). The origins of sex differences in human behavior: evolved dispositions versus social roles. American Psychologist, 54 (6), 408-423.

Fitch, R. H., Miller, S., \& Tallal, P. (1997). Neurobiology of speech perception. Annual Review Neuroscience, 20, 331-353.

Flores-Mendoza, C. (2000). Diferenças intelectuais entre homens e mulheres: uma breve revisão da literatura. Psicólogo inFormação, 4 (4), 25-34.

Forgus, R. H. (1971). Percepção: o processo básico do desenvolvimento cognitivo. São Paulo: Edusp.

Gil-Verora, J. A., Macías, J. A., Pastor, J. F., Paz. F., Barbosa, M., Maniega, M. A. et al. (2003). Diferencias sexuales en el sistema nervioso humano. Una revisón desde el punto de vista psiconeurobiológico. International Journal of Clinical and Health Psychology, 3 (2), 351-361.

Hampson, E., \& Kimura, D. (1993). Sex differences and hormonal influences on cognitive function in humans. In J. B. Becker, S. M. Breedlove, D. Crews \& M. M. McCarthy (Eds.), Behavioralendocrinology (pp.358-398). Cambridge, Mass: MIT Press.

Jongman, A., Sereno, J. A., \& Wang, Y. (2001). Dichotic perception of mandarin tones by Chinese and American listeners. Brain and Language, 78, 332-348. 
Kandel, E. R., Schwartz, J.H., \& Jessell, T.M. (1995) Fundamentos de neurociências e do comportamento. Rio de Janeiro: Guanabara Koogan.

Leontiev, A. N. (1960). On the biological and social aspects of human development: the training of auditory ability. In A. N. Leontiev, A. V. Zaporozhets (Eds.), Rehabilitation of hand function (pp.423-440). New York: Pergamon Press.

Matos, M. A. (1981). O controle de estímulos sobre o comportamento. Psicologia, 7 (2), 1-15.

Nater, U. M., Abbruzzese, E., Krebs, M., \& Ehlert, U. (2006). Sex differences in emotional and psychophysiological responses to musical stimuli. International Journal of Psychophysiology, 62, 300-308.

Rohden, F. (2003). A construção da diferença sexual na medicina. Cadernos de Saúde Pública, 19 (2), S201-S212.

Rosa e Silva, A. C. J. S. R., \& Sá, M. F. S. (2006) Efeitos dos esteróides sexuais sobre o humor e a cognição. Revista de Psiquiatria Clínica, 2 (33), 60-67.
Roth, I., \& Frisby, J. P. (1986). Perception and representation: a cognitive approach. Milton Keynes, UK: Open University.

Sanders, G., \&Wenmoth, D. (1998). Verbal and music dichotic listening tasks reveal variations in functional cerebral asymmetry across the menstrual cycle that are phase and task dependent. Neuropsychologia, 36 (9), 869-874.

Shamma, S. (1999). A physiological basis of timbre perception. In: M. Gazzaniga (Eds.), The new cognitive neurosciences (pp.411-450). Cambrigde, Mass: MIT Press.

Sluming, V. A., \& Manning, J. T. (2000). Second to fourth digit ratio in elite musicians: evidence for musical hability as an honest signal of male fitness. Evolution and Human Behavior, 21, 1-9.

Recebido em: 24/4/2007

Versão final reapresentada em: 10/12/2007

Aprovado em: 17/1/2008 